\title{
Translational Developmental Toxicology: Prospects for Protective Therapeutic Obstetrical and Neonatal Interventions
}

\author{
Claude Hughes $^{1 *}$, Michael Waters ${ }^{2}$, lyabo Obasanjo ${ }^{3}$ and David Allen ${ }^{2}$ \\ ${ }^{1}$ Quintiles Inc., Wake Forest University, Duke University Medical Center, USA \\ ${ }^{2}$ Integrated Laboratory Systems, Inc, USA \\ ${ }^{3}$ Advanced Leadership Fellow, Harvard University, US ${ }^{A}$
}

\begin{abstract}
Contemporary translational biomedical research strives to move from discoveries in models (in silico, in vitro and in vivo) into human clinical trials to expeditiously develop specific therapeutics. Translational toxicology must similarly strive to identify applicable therapeutics that can safely and effectively mitigate potential harm from exposures. Since human exposures to chemicals, physical agents and social factors are inevitable, the human fetus is subject to effects that can have lifelong consequences. In order to apply the translational concept to developmental toxicology, we are guided by the modest number of established and accepted obstetrical therapeutics used primarily for fetal benefit. These established or potential therapeutic obstetrical interventions suggest that early steps into testing or implementing developmental translational toxicology therapies during the in utero and early neonatal period will likely derive from Generally-Recognized-As-Safe (GRAS) options. If we are to translate environmental health discoveries into safe and effective interventions, we must assert and characterize valid, applicable therapies such as GRAS treatments and eventually "ethical pharmaceuticals" for the protective care of these highly vulnerable young patients. We can create a safe and efficacious environmental health portfolio of interventional options to improve human health that include both reduction/avoidance of exposure and specific preventative/mitigative/restorative therapeutics.
\end{abstract}

Keywords: Fetal exposures; Fetal therapy; Translational toxicology

\section{Introduction}

We have recently asserted that the relatively new term of "translational toxicology" should encompass both the existing principles of toxicology and epidemiology but be driven by the aim of developing safe and effective interventions to include prevention, mitigation or reversion of adverse human health effects of exposures. Exposure assessment, basic toxicology and development of certain categories of mathematical models to characterize exposure-outcome relationships are not new areas of research. However, overtly integrating these research processes in order to conceive, assess and validate ethical interventions to prevent, mitigate or reverse adverse effects of environmental exposures is the novel opportunity. The research and development aims within translational toxicology should be the creation of a safe and effective portfolio of interventional options to improve human health that goes beyond mere reduction and/or avoidance of exposure(s) so that practitioners have specific preventative/mitigative/ restorative therapeutics for human clinical use.

All environmental health research including developmental toxicology, is undoubtedly more challenging than conducting randomized controlled clinical trials. In environmental health research, we cannot have control over several key components in the "usual" human clinical trial research setting. Key characteristics of both the exposure (the agent, dose, duration, route, etc.) and the exposed subject (person's age, sex, developmental life-stage, inter-current illnesses, nutritional status, etc.) are not controlled by the investigator.

In general, contemporary translational research in all areas of medicine relies upon identification, validation and use of various biomarkers for execution of research and development programs. In human health research, the outcomes that truly matter are clinical endpoints (how a patient feels, functions or survives). Short of directly measuring clinical endpoints, surrogate endpoints can be measured, but at a minimum, biomarkers must be explicitly validated as predictors of surrogate endpoints to be plausibly interpreted as indicators of clinical endpoints. The validity of such linkage for many (novel) biomarkers can be challenged, but broad scientific-medical agreement can be reached for many others.

To simply illustrate the extreme challenges for use of biomarker tests in human health, we can contrast two well-known tests, namely Hemoglobin A1c (HbA1c) and Prostate Specific Antigen (PSA). HbA1c is a validated biomarker (in terms of clinical, regulatory, diagnostics and care) for the dysglycemia of type 2 diabetes mellitus (clinical endpoint). In contrast, PSA is a widely used biomarker that has only a modest degree of predictability for the surrogate endpoint of prostate histopathology, which in turn has only modest predictivity for clinically meaningful (i.e., life-threatening) forms of prostate cancer. The point is that we all must use any biomarkers with our eyes "wide open" so that we take advantage of these useful tools but do not leap to unjustified ultimate (clinical) conclusions.

For environmental health research, where the aim is to assess possible human health effects of exogenous exposures, how can we approximate a thorough validation process of linking biomarker(s) to surrogate endpoint(s) to clinical endpoint(s)? We must transform the usual logic of "translational" health research into an approach that "works" to move toxicology research into actionable environmental health discoveries. To plausibly establish in humans a causal relationship

*Corresponding author: Claude Hughes, Cardiovascular \& Metabolic Unit, Quintiles, Inc., Research Triangle Park, North Carolina 27709, USA, Tel: (+1) 919649-3920, (O): (+1) 919-998-7444; E-mail: claude.hughes@quintiles.com

Received October 27, 2013; Accepted November 28, 2013; Published November 28, 2013

Citation: Hughes C, Waters M, Obasanjo I, Allen D (2013) Translational Developmental Toxicology: Prospects for Protective Therapeutic Obstetrical and Neonatal Interventions. J Neonatal Biol 2: 122. doi:10.4172/2167-0897.1000122

Copyright: $\odot 2013$ Hughes $C$, et al. This is an open-access article distributed under the terms of the Creative Commons Attribution License, which permits unrestricted use, distribution, and reproduction in any medium, provided the original author and source are credited. 
between exposure and effect plus development and validation of safe and efficacious preventative/mitigative/restorative therapeutics in humans, we assert that the following clinical study design elements must be included in translational developmental toxicology research and development projects:

1. Indicator(s) of timing of exposure

2. Biomarker(s) of current exposure

3. Molecular/cellular biomarker(s) of systemic effects

4. Imaging biomarker(s) of target tissue effects

5. Molecular biomarkers of target tissue effects

6. Surrogate clinical outcomes

7. Clinical outcomes

8. Public health outcomes

Without such a spectrum of demonstrably linked, biologically plausible observations, mitigation strategies are difficult to propose and probably unethical to implement. With such a set of observations, mitigation, compensatory or therapeutic strategies can be conceived, and ethical interventional clinical trials can be designed and conducted.

An abiding challenge is to find some rational basis for narrowing the scientific focus in translational toxicology/translational environmental health. We simply cannot do everything for every possible exposure scenario, so how can we define a manageable scope of research themes with the greatest promise of translating discovery into clinical interventions? The long-standing concept in developmental toxicology is that there are critical life stages or certain key intervals in the lifespan which are Windows of Susceptibility (of adverse effects due to an exposure). The same biological reasoning that these critical life stages are particularly susceptible to adverse perturbation should mean that these same key intervals are likely also to be prime targets for intervention which we have called "Windows of Responsivity" [1], to minimize, negate or reverse antecedent or ongoing exposure effects. The preeminent category of exposed patients that should be addressed via the iterative process of translational toxicology research, advanced modeling and then testing of preventative/mitigative/restorative therapeutic interventions is the exposed gravid woman/mother-infant pairs (the early development window).

While "Fetal therapy is broadly defined as any intervention administered to or via the mother with a primary indication to improve perinatal or long-term outcomes for the fetus or newborn" [2], almost reflexively due to concern about unknown consequences of fetal exposures, the "precautionary principle" is generally assumed to be applicable regarding exposures of gravid women to exogenous agents of diverse types [1]. Nevertheless, the questions at hand are whether

a. there is already a background of broadly accepted dietary/ nutritional or pharmaceutical interventions that are given to pregnant women for the purpose of enhancing lifelong wellbeing of the mother's offspring, and

b. these can be taken as precedence for obstetrical or neonatal therapies that could be developed to obviate effects of developmental toxicants.

Beyond immunizations, care of maternal acute and chronic illnesses and specific obstetrical conditions (e.g, pre-eclampsia, gestational diabetes, etc.) including antenatal corticosteroids to enhance fetal lung maturity when premature delivery is anticipated, there are at least five different dietary/nutritional or pharmacological treatments that are commonly given to pregnant women where a primary aim is to effect fetal benefit that will obviously or likely manifest across the later lifespan.

\section{Established Therapeutic Obstetrical Intervention- Folate for Reduction in Risk of Neural Tube Defects}

It is universally accepted that pregnant women should have diets that are replete with folate (folic acid) with supplementation indicated as needed to reduce the risk of neural tube defects on the offspring [3,4]. In brief, the US Preventive Services Task Force recommendation is that all women planning or capable of becoming pregnant consume 0.4 to $0.8 \mathrm{mg}$ ( 400 to $800 \mu \mathrm{g}$ ) of folic acid daily to prevent Neural Tube Defects (NTDs). Studies have demonstrated that periconceptional folic acid supplementation can prevent $50 \%$ or more of NTDs such as spina bifida and anencephaly. Implementation of these recommendations is essential for the primary prevention of these serious and disabling birth defects. Because many reproductive age women do not consume the recommended amount of folic acid, there is an ongoing need to broadly encourage this intervention to minimize the risk of NTDs.

\section{Established Therapeutic Obstetrical and Neonatal Intervention- DHA for Vision and Cognitive Function}

The fetus and neonate should receive Long-Chain Polyunsaturated Fatty Acids (LC-PUFA) in amounts sufficient to support optimal visual and cognitive development. Moreover, the consumption of oils rich in n-3 LC-PUFA during pregnancy reduces the risk for early premature birth. Pregnant and lactating women should aim to achieve an average daily intake of at least $200 \mathrm{mg}$ DHA. For healthy term infants, Koletzko et al. [5] recommend and fully endorsed breastfeeding, which supplies preformed LC-PUFA, as the preferred method of feeding. When breastfeeding is not possible, they recommended use of an infant formula providing DHA at levels between 0.2 and 0.5 weight percent of total fat, and with the minimum amount of AA equivalent to the contents of DHA. In this context, pharmaceutical companies have responded by producing prenatal vitamin products that provide supplementation consistent with such recommendations for DHA intake during pregnancy.

\section{Potential Treatment of Pregnant Women for Fetal Benefit - Thyroid Hormone for Hypothyroidism during Gestation}

It is widely appreciated that avoiding maternal (and fetal) hypothyroidism during gestation is of major importance because of potential damage to fetal neural development, an increased incidence of miscarriage, and preterm delivery [6,7]. Management of thyroid diseases during pregnancy requires special considerations because pregnancy induces major changes in thyroid function and gestational age-specific values should be used for diagnosis and monitoring of therapy. Universal screening of pregnant women for thyroid disease is not yet supported by adequate studies, but the clinical standard-of-care to target specific groups of patients who are at increased risk is strongly supported [6,7].

Shields et al. [8] hypothesized that, even in the absence of overt thyroid dysfunction, maternal and fetal thyroid function influences fetal growth. They studied 616 healthy mother-child pairs by measuring TSH, free T4 (FT4), and Free T3 (FT3) in mothers at 28 wk gestation and in umbilical cord blood at birth. Birth weight, length, head 
circumference, and tricep and bicep skinfold thickness was measured on the babies. Associations between cord FT4 and birth size suggest that fetal thyroid function may be important in regulating fetal growth, both of skeletal size and fat. The correlation between third-trimester maternal FT4 and cord FT4 supports the belief that maternal T4 crosses the placenta even in late gestation.

Kuppens et al. [9] conducted a prospective follow-up study during three trimesters of gestation in 886 Dutch Caucasian healthy pregnant women followed from 12-week gestation until term delivery $(>37$ weeks) and their neonates. Maternal thyroid function during gestation was related to neonatal TT4 at screening.

An abiding concern has been that unrecognized mildly reduced thyroid hormone levels in pregnant women might adversely affect cognitive function of their offspring. Lazarus et al. [10] conducted a randomized trial in which pregnant women at a gestation of 15 weeks 6 days or less provided blood samples for measurement of thyrotropin and free thyroxine (T4). The primary outcome was IQ at 3 years of age in children of women with positive results, as measured by psychologists who were unaware of the group assignments. Of 21,846 women who provided blood samples (at a median gestational age of 12 weeks 3 days), 390 women in the screening group and 404 in the control group tested positive. Antenatal screening (at a median gestational age of 12 weeks 3 days) and maternal treatment for hypothyroidism did not result in improved cognitive function in children at 3 years of age.

\section{Potential Treatment of Pregnant Women for Fetal Benefit - $\mathrm{MgSO}_{4}$ for Reduction in Risk of Cerebral Palsy}

Nelson and Grether [11] observed that in utero exposure to magnesium sulfate $\left(\mathrm{MgSO}_{4}\right)$ was associated with a lower prevalence of cerebral palsy (CP) in infants weighing $<1500 \mathrm{~g}$ at birth. In a randomized controlled study, Rouse et al. [12] found that fetal exposure to magnesium sulfate before anticipated early preterm delivery did not reduce the combined risk of moderate or severe cerebral palsy or death, although the rate of cerebral palsy was reduced among survivors.

Conde-Agudelo and Romero [13] performed a systematic review and meta-analysis of randomized controlled trials to determine whether magnesium sulfate administered to women at risk of preterm delivery before 34 weeks of gestation may reduce the risk of cerebral palsy in their children and concluded magnesium sulfate administered to women at risk of delivery before 34 weeks of gestation reduces the risk of cerebral palsy.

Rouse [14] argued that on the basis of three large, randomized placebo-controlled trials of antenatal magnesium sulfate $\left(\mathrm{MgSO}_{4}\right)$ for fetal neuroprotection, there is strong support for the utilization of $\mathrm{MgSO}_{4}$ to lower the risk of cerebral palsy among the survivors of early preterm birth. In the United States, the use of $\mathrm{MgSO}_{4}$ for fetal neuroprotection has the potential to prevent 1000 cases of handicapping cerebral palsy annually [14].

Doyle et al. [15] also systematically reviewed rates of neurologic outcomes reported in childhood for the preterm fetus exposed to antenatal magnesium sulfate. These investigators searched the Cochrane Pregnancy and Childbirth Group's Trials Register, CENTRAL (The Cochrane Library 2008, Issue 3), relevant references from retrieved articles, and abstracts submitted to major congresses. The overall conclusion was that antenatal magnesium sulfate therapy given to women at risk of preterm birth is neuroprotective against motor disorders in childhood for the preterm fetus.

\section{Potential Treatment of Pregnant Women for Fetal Benefit - Nicotine or N-Acetyl-L-Cysteine (NAC) for Smoking}

Nicotine-replacement therapy is effective for smoking cessation outside pregnancy and even though its safety has not been thoroughly established, its use is widely recommended during pregnancy.

Coleman et al. [16] found no significant difference in the rate of abstinence from the quit date until delivery between the nicotinereplacement and placebo groups $(9.4 \%$ and $7.6 \%$, respectively; unadjusted odds ratio with nicotine-replacement therapy, 1.26 ; $95 \%$ confidence interval, 0.82 to 1.96 ). Compliance was low; only $7.2 \%$ of women assigned to nicotine-replacement therapy and $2.8 \%$ assigned to placebo used patches for more than 1 month. Rates of adverse pregnancy and birth outcomes were similar in the two groups. Adding a nicotine patch ( $15 \mathrm{mg}$ per 16 hours) to behavioral cessation support for women who smoked during pregnancy did not significantly increase the rate of abstinence from smoking until delivery or the risk of adverse pregnancy or birth outcomes.

Although smoking cessation is the primary goal for the control of cancer and other smoking-related diseases, chemoprevention provides a complementary approach applicable to high risk individuals such as current smokers and ex-smokers [17]. On the whole, there is overwhelming evidence that N-Acetyl-L-Cysteine (NAC) has the ability to modulate a variety of DNA damage- and cancer-related end-points.

While we do not know of studies in pregnant women, Van Schooten et al. [18] studied NAC in healthy smoking volunteers in a randomized, double-blind, placebo-controlled, Phase II chemoprevention trial. The subjects were supplemented daily with $2 \times 600 \mathrm{mg}$ of oral tablets of NAC $(n=20)$ or placebo $(n=21)$ for a period of 6 months, and internal dose markers [plasma and Bronchoalveolar Lavage (BAL) fluid cotinine, urine mutagenicity], biologically effective dose markers [smokingrelated DNA adducts and Hemoglobin $(\mathrm{Hb})$ adducts], and biological response markers (micronuclei frequency and antioxidants scavenging capacity) were assessed at both pre- and post-supplementation times ( $\mathrm{T}_{0}$ and $\mathrm{T}_{1}$ respectively). Overall, the internal dose markers remained unchanged at $\mathrm{T}_{1}$ as compared with $\mathrm{T}_{0}$ in both NAC and placebo groups. When quantifying the biologically effective dose markers, data showed an inhibitory effect of NAC toward the formation of lipophilic-DNA adducts $(5.18 \pm 0.73$ versus $4.08 \pm 1.03 / 108$ nucleotides; mean $\pm \mathrm{SE}$; $\mathrm{P}=0.05)$ as well as of 7,8-dihydro-8-oxo-2-eoxyguanosine adducts in BAL cells $(3.9 \pm 0.6$ versus $2.3 \pm 0.2 / 105$ nucleotides; $\mathrm{P}=0.003)$. There was no effect of NAC on the formation of lipophilic-DNA adducts in peripheral blood lymphocytes. Based on these results, the investigators concluded that NAC has the potential to alter tobacco smoke carcinogenicity in adult humans because it can modulate certain cancer associated biomarkers in specific organs, and by implication could mitigate DNA damage in the fetus.

While women are routinely advised to stop smoking (and most do), many non-smoking pregnant women are exposed to second hand smoke that can be detected by the biomarker cotinine in urine samples of pregnant women [19]. Therefore, this biomarker could be used as a guide for NAC intervention in pregnancy as a possible way to reduce harm to the fetus.

Potential Future “GRAS" (Generally-Recognized-AsSafe) Interventions for Exposures to Developmental Toxicants

Potential therapeutics to mitigate the adverse effects of toxic 
exposures is likely to come from investigation of compounds that are acknowledged as "GRAS", generally-recognized-as-safe with several different Modes-of-Action (MOA). Multiple potential modes of action will need to be assessed; safety profiles will need to be developed; and there is minimal expectation that commercial entities will be motivated to make the investments required to develop agents to pre-empt or reduce adverse outcomes later in life due to antecedent environmental exposures. Logical starting points are to consider instances where clinical practice already uses a protective treatment to reduce the risk of acute toxicity and the insights gained from work done in the area of chemoprevention of cancer(s).

\section{General MOAs- Possibilities for Further Exploration of Maternal-Fetal Effects}

Increasing attention is being paid to the possibility of applying cancer chemopreventive agents for individuals at high risk of neoplasia. For this purpose, natural compounds have practical advantages with regard to availability, suitability for oral application, regulatory approval and mechanisms of action [20]. Candidate substances such as phytochemicals present in foods and their derivatives have been identified by a combination of epidemiological and experimental studies. Plant constituents include vitamin derivatives, phenolic and flavonoid agents, organic sulfur compounds, isothiocyanates, curcumins, fatty acids and d-limonene. Examples of compounds from animals are unsaturated fatty acids and lactoferrin. Recent studies have indicated that mechanisms underlying chemopreventive potential may be combinations of anti-oxidant, anti-inflammatory, immune-enhancing, and anti-hormone effects, with modification of drug-metabolizing enzymes, influence on the cell cycle and cell differentiation, induction of apoptosis and suppression of proliferation and angiogenesis playing roles in the initiation and secondary modification stages of neoplastic development.

Murphy et al. [21] estimated usual intakes of nine individual phytonutrients by Americans consuming recommended levels of fruits and vegetables compared to intakes by adults not meeting these recommendations, and sought to identify contributions of food sources to total phytonutrient intakes. The phytonutrients examined in this study are found predominantly in fruits and vegetables. Food consumption data from the National Health and Nutrition Examination Surveys 2003-2006 and phytonutrient concentration data from US Department of Agriculture databases and the published literature were used to estimate energy-adjusted usual intakes. Mean energy-adjusted phytonutrient intakes were compared between subpopulations who consumed recommended amounts of fruits and vegetables versus those who did not. Percentage contributions of each phytonutrient by food source were estimated for all adults. Energy-adjusted intakes of all phytonutrients other than ellagic acid were considerably higher among both men and women meeting dietary recommendations for fruit and vegetable intakes compared to those not meeting the recommendations; energy-adjusted intakes of ellagic acid were higher only among women meeting versus not meeting the recommendations. For five of the nine phytonutrients ( $\alpha$-carotene, $\beta$-cryptoxanthin, lycopene, hesperetin, and ellagic acid), a single food typically accounted for $64 \%$ or more of the total intake of the phytonutrient. Energy-adjusted intakes of carotenoids and flavonoids are higher among men and women whose diets conform to dietary guidance for fruits and vegetables. A limited number of foods provide the majority of these phytonutrients. Findings from this research provide important reference information on the phytonutrient contributions of a diet rich in fruits and vegetables.
A key technical challenge in assessing exposures is to measure dietary intake of various foods in the population at large. Labonte' et al. [22] assessed the validity and the reproducibility of a newly developed web-based, self-administered Food Frequency Questionnaire (webFFQ). A total of 74 healthy subjects ( 34 men and 40 women) from the Quebec City metropolitan area were asked to complete, in random order, the web-FFQ, a validated interviewer-administered FFQ (IAFFQ) and a 3-day food record (3-day FR). The data demonstrate that the web-based FFQ appears to have reasonable validity and good reproducibility for assessing nutrient intakes at the group and individual levels in a population of healthy adults. Thus, there is the potential of using dietary intake of phytonutrients from web-FFQ in pregnant women to assess exposures to these classes of compounds and potentially fetal and infant outcomes.

\section{Possibilities for Further Exploration of Maternal-Fetal Effects - NAC to Protect Kidneys in Smokers/Passive Smokers}

Although the evidence of efficacy is somewhat mixed, NAC is widely used to reduce the risk of acute contrast nephrotoxicity in patients with impaired renal function who undergo computed tomography scanning. To determine whether oral NAC protects against deterioration in renal function in patients with moderate renal insufficiency who undergo elective coronary angiography, Kay et al. [23] conducted a prospective, randomized, double-blind, placebocontrolled trial in 200 patients aged mean (SD) 68 (6.5) years with stable moderate renal insufficiency (creatinine clearance $<60 \mathrm{~mL} / \mathrm{min}$ $[1.00 \mathrm{~mL} / \mathrm{s}]$ ) who were undergoing elective coronary angiography with or without intervention. Participants were randomly assigned to receive oral NAC (600 mg twice per day; $\mathrm{n}=102$ ) or matching placebo tablets $(n=98)$ on the day before and the day of angiography. All patients received low-osmolality contrast agent. NAC treatment significantly increased creatinine clearance from $44.8 \mathrm{~mL} / \mathrm{min}(0.75 \mathrm{~mL} / \mathrm{s})(95 \% \mathrm{CI}$, $42.7-47.6 \mathrm{~mL} / \mathrm{min})$ to $58.9 \mathrm{~mL} / \mathrm{min}(0.98 \mathrm{~mL} / \mathrm{s})(95 \% \mathrm{CI}, 55.6-62.3 \mathrm{~mL} /$ min) 2 days after the contrast administration $(\mathrm{P}=0.001)$. The increase was not significant in the control group (from 42.1 to $44.1 \mathrm{~mL} / \mathrm{min}$ [0.70 to $0.74 \mathrm{~mL} / \mathrm{s}] ; \mathrm{P}=.15$ ). The benefit of NAC was consistent among various patient subgroups and persistent for at least 7 days. There were no major treatment-related adverse events. The investigators concluded that NAC protects patients with moderate chronic renal insufficiency from contrast-induced deterioration in renal function after coronary angiographic procedures, with minimal adverse effects and at a low cost.

\section{Possibilities for Further Exploration of Maternal-Fetal Effects - HDAC Inhibitors}

The reversible acetylation of histones is an important mechanism of gene regulation. During prostate cancer progression, specific modifications in acetylation patterns on histones are apparent. Targeting the epigenome, including the use of Histone Deacetylase (HDAC) inhibitors, is a novel strategy for cancer chemoprevention. Recently, drugs classified as HDAC inhibitors have shown promise in cancer clinical trials. Ho et al. [24] have shown that Sulforaphane (SFN), a compound found in cruciferous vegetables, inhibits HDAC activity in human colorectal and prostate cancer cells. Based on the similarity of SFN metabolites and other phytochemicals to known HDAC inhibitors, they found that sulforaphane acted as an HDAC inhibitor in the prostate, causing enhanced histone acetylation, derepression of P21 and Bax, and induction of cell cycle arrest/apoptosis, leading to cancer prevention. The ability of SFN to target aberrant acetylation patterns, 
in addition to effects on phase 2 enzymes, may make it an effective chemoprevention agent. Their studies suggest that high-risk prostate cancer patients might increase survival through simple dietary choices that incorporate readily accessible foods into their diets.

\section{Possibilities for Further Exploration of Maternal-Fetal Effects - Reduction of ROS Formation}

Harms-Ringdahl et al. [25] conducted a study to address whether tomato juice protects against formation of Reactive Oxygen Species (ROS) induced by extensive physical exercise in untrained individuals. As a marker of oxidative stress, serum levels of 8-oxodG were monitored using a modified ELISA. An intervention was performed involving 15 untrained healthy subjects who performed a 20 min physical exercise at $80 \%$ of maximum pulse using an ergometer bicycle. Blood samples were taken before and one hour after the exercise. The procedure was repeated after 5 weeks with a daily intake of $150 \mathrm{ml}$ tomato juice and followed by a 5 weeks wash-out period and another 5 weeks with a daily intake of tomato juice. The results indicated that a daily intake of tomato juice, equal to $15 \mathrm{mg}$ lycopene per day, for 5 weeks significantly reduced the serum levels of 8-oxodG after an extensive physical exercise.

Their data suggest that tomato juice has a potential antioxidant effect and may reduce the elevated level of ROS induced by oxidative stress. Since DNA, lipids and proteins are constantly exposed to Reactive Oxygen Species (ROS), the molecular injury due to ROS exposure may be reduced by this dietary intervention.

\section{Possibilities for Further Exploration of Maternal-Fetal Effects - Uncertain MOA but Antidiabetogenic Actions of Epigallocatechin Gallate (EGCG)}

Green tea was suggested as a therapeutic agent for the treatment of diabetes more than 70 years ago, but the mechanisms behind its antidiabetic effect remains elusive. Ortsäter et al. [26] addressed this issue by feeding a green tea extract (TEAVIGO ${ }^{\mathrm{m}}$ ) with a high content of Epigallocatechin Gallate (EGCG) or the thiazolidinedione PPAR- $\gamma$ agonist rosiglitazone, as positive control, to $\mathrm{db} / \mathrm{db}$ mice, an animal model for diabetes. Young ( 7 week-old) $\mathrm{db} / \mathrm{db}$ mice were randomized and assigned to receive diets supplemented with or without EGCG or rosiglitazone for 10 weeks. Fasting blood glucose, body weight and food intake was measured along the treatment. Glucose and insulin levels were determined during an oral glucose tolerance test after 10 weeks of treatment. Pancreata were sampled at the end of the study for blinded histomorphometric analysis. Islets were isolated and their mRNA expression analyzed by quantitative RT-PCR. The results show that, in $\mathrm{db} / \mathrm{db}$ mice, EGCG improves glucose tolerance and increases glucosestimulated insulin secretion. EGCG supplementation reduces the number of pathologically changed islets of Langerhans, increases the number and the size of islets, and heightens pancreatic endocrine area. These effects occurred in parallel with a reduction in islet endoplasmic reticulum stress markers, possibly linked to the antioxidative capacity of EGCG. Their data show that the green tea extract EGCG markedly preserves islet structure and enhances glucose tolerance in genetically diabetic mice. Dietary supplementation with EGCG could potentially contribute to nutritional strategies for the prevention and treatment of type 2 diabetes.

\section{Possibilities for Further Exploration of Maternal-Fetal Effects-Polyphenols with Multiple MOAs}

Resveratrol, a polyphenol in red wine, has been reported as a calorie restriction mimetic with potential antiaging and antidiabetogenic properties. Park et al. [27] found that the metabolic effects of resveratrol result from competitive inhibition of cAMPdegrading phosphodiesterases, leading to elevated cAMP levels. The resulting activation of Epac1, a cAMP effector protein, increases intracellular $\mathrm{Ca} 2+$ levels and activates the CamKK $\beta$-AMPK pathway via phospholipase $\mathrm{C}$ and the ryanodine receptor $\mathrm{Ca} 2+-$ release channel. As a consequence, resveratrol increases NAD+ and the activity of Sirt1. Inhibiting PDE4 with rolipram reproduces all of the metabolic benefits of resveratrol, including prevention of diet-induced obesity and an increase in mitochondrial function, physical stamina, and glucose tolerance in mice. Therefore, administration of PDE4 inhibitors may also protect against and ameliorate the symptoms of metabolic diseases associated with aging.

The search for complementary treatments in primary prevention of Cardiovascular Disease (CVD) is a high-priority challenge. Grape and wine polyphenol resveratrol confers $\mathrm{CV}$ benefits, in part by exerting anti-inflammatory effects. However, the evidence in human long-term clinical trials has yet to be established. Tomé-Carneiro et al. [28] investigated the effects of a dietary resveratrol-rich grape supplement on the inflammatory and fibrinolytic status of subjects at high risk of CVD and treated according to current guidelines for primary prevention of CVD. Seventy-five patients undergoing primary prevention of CVD participated in this triple-blinded, randomized, parallel, dose-response, placebo-controlled, 1-year follow-up trial. Patients, allocated in 3 groups, consumed placebo (maltodextrin), a resveratrol-rich grape supplement (resveratrol $8 \mathrm{mg}$ ), or a conventional grape supplement lacking resveratrol, for the first 6 months and a double dose for the next 6 months. In contrast to placebo and conventional grape supplement, the resveratrol-rich grape supplement significantly decreased high-sensitivity C-reactive protein $(-26 \%, \mathrm{p}=0.03)$, tumor necrosis factor- $\alpha(-19.8 \%, p=0.01)$, plasminogen activator inhibitor type $1(-16.8 \%, \mathrm{p}=0.03)$, and interleukin-6/interleukin-10 ratio $(-24 \%, p=0.04)$ and increased anti-inflammatory interleukin-10 $(19.8 \%, \mathrm{p}=0.00)$. Adiponectin $(6.5 \%, \mathrm{p}=0.07)$ and soluble intercellular adhesion molecule- $1(-5.7 \%, p=0.06)$ tended to increase and decrease, respectively. No adverse effects were observed in any patient. In conclusion, 1-year consumption of a resveratrol-rich grape supplement improved the inflammatory and fibrinolytic status in patients who were on statins for primary prevention of CVD and at high CVD risk (i.e., with diabetes or hypercholesterolemia plus $\geq 1$ other CV risk factor). Their results show that a dietary intervention with grape resveratrol could complement the gold standard therapy in the primary prevention of CVD.

\section{Possibilities for Further Exploration of Maternal-Fetal Effects - The Complexity of Carotenoids with Multiple MOAs}

Clinical outcomes of dietary trials of large doses of carotenoids have not shown consistent results. Eroglu et al. [29] have developed a potential explanation based on studies of metabolism of $\beta$-carotene, the major dietary source of provitamin A. Central cleavage of $\beta$-carotene catalyzed by $\beta$-carotene oxygenase 1 yields two molecules of retinaldehyde. Subsequent oxidation produces All-Trans-Retinoic Acid (ATRA), which functions as a ligand for a family of nuclear transcription factors, the Retinoic Acid Receptors (RARs). Eccentric cleavage of $\beta$-carotene at non-central double bonds is catalyzed by other enzymes and can also occur non-enzymatically. The products of these reactions are $\beta$-apocarotenals and $\beta$-apocarotenones, whose biological functions in mammals are unknown. The investigators used reporter gene assays 
to show that none of the $\beta$-apocarotenoids significantly activated RARs. Importantly, however, $\beta$-apo-14'-carotenal, $\beta$-apo-14'-carotenoic acid, and $\beta$-apo-13-carotenone antagonized ATRA-induced transactivation of RARs. Competitive radioligand binding assays demonstrated that these putative RAR antagonists compete directly with retinoic acid for high affinity binding to purified receptors. Molecular modeling studies confirmed that $\beta$-apo-13-carotenone can interact directly with the ligand binding site of the retinoid receptors. $\beta$-Apo-13-carotenone and the $\beta$-apo-14'-carotenoids inhibited ATRA-induced expression of retinoid responsive genes in Hep G2 cells. An LC/MS method was developed and demonstrated that 3-5 nM $\beta$-apo-13-carotenone was present in human plasma. These findings suggest that $\beta$-apocarotenoids function as naturally occurring retinoid antagonists. The antagonism of retinoid signaling by these metabolites may have implications for the activities of dietary $\beta$-carotene as a provitamin $A$ and as a modulator of risk for cardiovascular disease and cancer.

\section{Possibilities for Further Exploration of Maternal- Fetal Effects-Anastrazole to Sustain Development of Androgen-Responsive Tissues from Effects of Exposures to Environmental Antiandrogens}

Vinclozolin is an Endocrine-Disrupting Chemical (EDC) that binds with high affinity to the Androgen Receptor (AR) and blocks the action of gonadal hormones on male reproductive organs. An alternative mechanism of action of vinclozolin involves transgenerational effects on the male reproductive tract. Cowin et al. [30] postulated the male reproductive abnormalities induced by in utero vinclozolin exposure could be reversed by testosterone supplementation and thereby avoid the permanent modifications involving DNA methyltransferases (Dnmts) described by others. To test this hypothesis, the investigators administered high-dose testosterone at puberty to vinclozolin-treated rats and determined the effect on Anogenital Distance (AGD); testicular germ cell apoptosis, concentration of elongated spermatids, the onset of prostatitis and Dnmt1, $-3 \mathrm{~A},-3 \mathrm{~B}$, and $-3 \mathrm{~L}$ mRNA expression. Consistent with previous reports, in utero exposure to vinclozolin significantly reduced AGD, increased testicular germ cell apoptosis 3 -fold, reduced elongated spermatid number by $40 \%$, and induced postpubertal prostatitis in $100 \%$ of exposed males. Administration of high-dose testosterone $(25 \mathrm{mg} / \mathrm{kg}$ ) at puberty normalized AGD, reduced germ cell apoptosis, and restored elongated spermatid number. Testosterone restored $\mathrm{AR}$ and nuclear factor- $\kappa \mathrm{B}$ expression in the prostate and abolished vinclozolin-induced prostatitis. Altered Dnmt expression was evident with in utero vinclozolin exposure and was not normalized after testosterone treatment. These data demonstrate in utero vinclozolin-induced male reproductive tract abnormalities are AR mediated and reversible and involve a mechanism independent of Dnmt expression. As the field of translational developmental toxicology progresses, it is possible that an "ethical pharmaceutical" compound such as anastrazole could be considered for administration to attain submaximal inhibition of aromatase in the fetus thereby modestly increasing endogenous androgen production to compensate for antiandrogenic effects of environmental antiandrogen exposures.

\section{Possibilities for Further Exploration of Maternal-Fetal Effects - Resveratrol or 3,4-DMF as AhR Antagonists to Protect the Ovaries from Effects of Exposure to Smoking}

It has been shown that benzo[a]pyrene, a key component of cigarette smoke and an Aryl hydrocarbon Receptor (AhR) ligand, reduced growth of isolated rat follicles in vitro. However, the mechanism underlying the induced changes in folliculogenesis is unknown. Neal et al. [31] proposed that the reported adverse effects of benzo[a] pyrene on follicle growth are mediated through AhR activation. The objective was to investigate the effect of benzo[a]pyrene with and without AhR antagonists (resveratrol or 3',4'-dimethoxyflavone (3,4DMF)) on follicle growth, oestradiol output, Anti-Müllerian hormone $(\mathrm{AMH})$ concentration and cell proliferation in isolated rat follicles cultured in vitro. Benzo[a]pyrene treatment significantly inhibited follicle growth and cell proliferation at concentrations of $1.5 \mathrm{ng} / \mathrm{ml}$ and higher $(\mathrm{P}<0.05)$, an effect attenuated by co-incubation with benzo[a] pyrene and resveratrol or 3,4-DMF. A significant decrease in oestradiol $(\mathrm{P}<0.05)$ and $\mathrm{AMH}$ output $(\mathrm{P}<0.001)$ by cultured follicles was induced by benzo[a]pyrene treatment, an effect attenuated by co-incubation with 3,4-DMF. The results suggest that the adverse effects of benzo[a]pyrene on follicle growth, steroidogenesis and $\mathrm{AMH}$ output are mediated through activation of the AhR. Moreover, AhR antagonists such as resveratrol and 3,4-DMF may have therapeutic benefit in protecting the ovary against the adverse effects of AhR ligands, including benzo[a] pyrene.

\section{Possibilities for Further Exploration of Maternal-Fetal Effects - Optimal Vitamin D for Multiple Potential Target Tissue Benefits}

Mounting evidence suggests that vitamin D deficiency could be linked to several chronic diseases, including cardiovascular disease and cancer. Forrest and Stuhldreher [32] examined the prevalence of vitamin $\mathrm{D}$ deficiency and its correlates to test the hypothesis that vitamin D deficiency was common in the US population, especially in certain minority groups. The National Health and Nutrition Examination Survey 2005 to 2006 data were analyzed for vitamin D levels in adult participants $(\mathrm{N}=4495)$. Vitamin $\mathrm{D}$ deficiency was defined as a serum 25-hydroxyvitamin $\mathrm{D}$ concentrations $\leq 20 \mathrm{ng} / \mathrm{mL}(50$ $\mathrm{nmol} / \mathrm{L}$ ). The overall prevalence rate of vitamin $\mathrm{D}$ deficiency was $41.6 \%$, with the highest rate seen in blacks (82.1\%), and followed by Hispanics (69.2\%). Vitamin D deficiency was significantly more common among those who had no college education, were obese, with a poor health status, hypertension, low high-density lipoprotein cholesterol level, or not consuming milk daily (all $\mathrm{P}<.001$ ). Multivariate analyses showed that being from a non-white race, not college educated, obese, having low high-density lipoprotein cholesterol, poor health, and no daily milk consumption were all significantly, independently associated with vitamin $\mathrm{D}$ deficiency (all $\mathrm{P}<.05$ ). In summary, vitamin $\mathrm{D}$ deficiency was common in the US population, especially among blacks and Hispanics. Given that vitamin D deficiency is linked to some of the important risk factors of leading causes of death in the United States, it is important that health professionals are aware of this connection and offer dietary and other intervention strategies to correct vitamin D deficiency, especially in minority groups.

Higher total serum 25-Hydroxyvitamin D (25(OH)D) concentrations have been associated with better cognitive function mainly in cross-sectional studies in adults. It is unknown if the associations of different forms of $25(\mathrm{OH}) \mathrm{D}(25(\mathrm{OH}) \mathrm{D} 3$ and $25(\mathrm{OH})$ D2) are similar. Tolppanen et al. [33] conducted a prospective cohort study $(\mathrm{n}=3171)$ with serum $25(\mathrm{OH}) \mathrm{D} 3$ and $25(\mathrm{OH}) \mathrm{D} 2$ concentrations measured at mean age of 9.8 years and academic performance at age 13-14 years (total scores in English, mathematics and science) and 15-16 years (performance in General Certificates of Education examinations). Serum 25(OH)D3 concentrations were not associated with any educational outcomes. Higher $25(\mathrm{OH}) \mathrm{D} 2$ concentrations were associated with worse performance in English at age 13-14 years (adjusted SD change per doubling in $25(\mathrm{OH}) \mathrm{D} 2(95 \% \mathrm{CI})-0.05(-0.08$ 
to -0.01$)$ ) and with worse academic performance at age 15-16 years (adjusted OR for obtaining $\geq 5 \mathrm{~A}^{*}-\mathrm{C}$ grades (95\% CI) 0.91 (0.82 to $1.00)$ ).

The null findings with 25(OH)D3 are in line with two previous cross-sectional studies in children. It is possible that the positive association of $25(\mathrm{OH}) \mathrm{D}$ with cognitive function seen in adults does not emerge until later in life or that the results from previous crosssectional adult studies are due to reverse causality. The unexpected inverse association of 25(OH)D2 with academic performance requires replication in further studies. These investigators concluded that their results did not support suggestions that children should have controlled exposure to sunlight, or vitamin D supplements, in order to increase academic performance.

Previous studies indicate reduced risk of type 1 diabetes after intake of vitamin D supplements during pregnancy or early childhood. Sørensen et al. [34] studied whether lower maternal serum concentrations of 25-hydroxy-vitamin D (25-OH D) during pregnancy were associated with an increased risk of childhood-onset type 1 diabetes. In this casecontrol study nested within a cohort of 29,072 women in Norway, 25$\mathrm{OH} \mathrm{D}$ levels were measured using a radioimmunoassay on samples from late pregnancy in 109 women delivering a child who developed type 1 diabetes before 15 years of age (case subjects) and from 219 control women. Dividing the levels of maternal 25-OH D into quartiles, there was a trend toward a higher risk of type 1 diabetes with lower levels of vitamin D during pregnancy. The odds of type 1 diabetes was more than twofold higher for the offspring of women with the lowest levels of 25$\mathrm{OH} \mathrm{D}$ compared with the offspring of those with levels above the upper quartile. The data provide support for the initiation of a randomized intervention trial to prevent type 1 diabetes in children by enhancing maternal 25-OH D status during pregnancy.

\section{Discussion}

Human exposures to chemicals, physical agents and social factors are inevitable. All too often the medical practitioner's view of toxicology and human health is the assumption that the old adage [1] of "Absence of evidence (of effect or harm) is not evidence of absence (of effect or harm)" can be ignored! To overcome that therapeutic nihilism, a simple but demanding challenge faces us. To optimize human health [1], all concerned stakeholders including regulators, commercial entities, health providers, investigators and the public at large need to know the following:

a) which exposures (dose, duration, window of exposure, other susceptibility factors, etc.) pose a risk that justifies

b) public health intervention(s) to reduce exposure or

c) individual health care therapeutic or mitigating treatments. If we are to translate our environmental health science (discovery) into action (safe and effective interventions), we must assert and show how this continuum of discovery will equip medical practitioners with valid, applicable therapies such as effective GRAS treatments and "ethical pharmaceuticals" for care of their patients.

In regards to the timing of interventions for prevention or mitigation, we do need to consider that for certain adverse effects/ disease predispositions due to environmental exposures, there may need to be sustained, continuing interventions. Nevertheless, key windows for interventions are likely to be the same as those in which adverse effects are elicited. Thus, in utero/neonatal/early childhood, adolescent/ peripubertal and then menopausal transition in women may be particularly responsive since organizational/re-organizational processes are biologically dynamic in those intervals [1].

\section{Summary: The Therapeutic Prospects}

We must get beyond the simplistic notion that the only method to reduce risk of adverse health outcomes from developmental exposures is to "avoid exposure". While this is not advocacy of a "go ahead and pollute" policy, we must acknowledge that there are different sorts of exposures. Some exposures are intentional (pharmaceutical drug use), some are elective (ethanol consumption, tobacco smoking, etc.) and some are quasi-elective (dietary choices); however many exposures of concern are truly unintended (air quality, water quality, workplace, some dietary contaminants) and essentially inevitable. Body burdens of persistent compounds won't "go away" tomorrow by making a decision about what you eat today. Particulates in the air are unavoidable depending on where you live. From ethical and public health perspectives, this category of unintended exposures merits the greatest attention for development of possible intervention/mitigation beyond the simplistic approach of "Just go and reduce your exposure." Therefore we need to ascertain how to translate results from research toxicology and epidemiology studies into interventions that can/ should plausibly mitigate adverse health effects of exposures. These interventions will almost certainly need to be GRAS (GenerallyRecognized-As-Safe) or will depend upon use of well-established pharmaceuticals. It seems implausible to speculate that private industry would commit to spending billions of dollars to develop a new chemical entity) for prevention or reduction of adverse health effects due to an environmental health exposure. Efforts within the National Cancer Institute to encourage development of cancer preventative therapies may serve as a prototype for prevention of other chronic diseases [35]. Chemoprevention research at the NCI has sought to identify potential agents that can reduce cancer risk and develop public health strategies to take advantage of basic research discoveries. Their experiences will likely provide "lessons learned" for future development of translational environmental health therapeutics. The critical window of the maternalfetal/neonatal interval is wide open for investigation of possible GRAS therapeutic interventions to reduce diseases in the later lifespan of the offspring.

For implementation of this work, we suggest that for U.S.-based research, the National Institute of Environmental Health Sciences Clinical Research Unit could be used to exclusively conduct some studies, but should also serve as the "lead site" for a clinical research alliance for investigators in obstetrics, pediatrics and other selected medical specialties for research programs requiring a greater scale than can be realistically managed at one location. Such an alliance with a modest network of clinical investigators (study sites) who practice obstetrics, perinatal medicine, neonatology and/or pediatrics, would be critical for assessing whether interventions derived from this exposureoutcome or exposure-effect paradigm could be truly put into medical practice.

\section{References}

1. Hughes C, Waters M, Allen D, Obasanjo I (2013) Translational toxicology: a developmental focus for integrated research strategies. BMC Pharmacol Toxicol 14: 51

2. Pauli JM, Repke JT (2013) Update: obstetrics. OBG Management 25: 28-31.

3. (1999) Folic acid for the prevention of neural tube defects. American Academy of Pediatrics. Committee on Genetics. Pediatrics 104: 325-327.

4. U.S. Preventive Services Task Force (2009) Folic acid for the prevention of neural tube defects: U.S. Preventive Services Task Force recommendation statement. Ann Intern Med 150: 626-631. 
Citation: Hughes C, Waters M, Obasanjo I, Allen D (2013) Translational Developmental Toxicology: Prospects for Protective Therapeutic Obstetrical and Neonatal Interventions. J Neonatal Biol 2: 122. doi:10.4172/2167-0897.1000122

5. Koletzko B, Lien E, Agostoni C, Böhles H, Campoy C, et al. (2008) The roles of long-chain polyunsaturated fatty acids in pregnancy, lactation and infancy: review of current knowledge and consensus recommendations. J Perinat Med 36: $5-14$

6. Abalovich M, Amino N, Barbour L, Cobin RH, De Groot LJ, et al. (2007) Management of thyroid dysfunction during pregnancy and postpartum: an Endocrine Society clinical practice guideline. J Clin Endocrinol Metab 92: S1S47.

7. Rivkees SA, Mandel SJ (2011) Thyroid disease in pregnancy. Horm Res Paediatr 76 Suppl 1: $91-96$

8. Shields BM, Knight BA, Hill A, Hattersley AT, Vaidya B (2011) Fetal thyroid hormone level at birth is associated with fetal growth. J Clin Endocrinol Metab 96: E934-938.

9. Kuppens SM, Kooistra L, Wijnen HA, Vader HL, Hasaart TH, et al. (2011) Neonatal thyroid screening results are related to gestational maternal thyroid function. Clin Endocrinol (Oxf) 75: 382-387.

10. Lazarus JH, Bestwick JP, Channon S, Paradice R, Maina A, et al. (2012) Antenatal thyroid screening and childhood cognitive function. $\mathrm{N}$ Engl $\mathrm{J}$ Med 366: 493-501.

11. Nelson KB, Grether JK (1995) Can magnesium sulfate reduce the risk of cerebral palsy in very low birthweight infants? Pediatrics 95: 263-269.

12. Rouse D, Hirtz D, Thom E, Varner M, Spong C, et al. (2008) A randomized, controlled trial of magnesium sulfate for the prevention of cerebral palsy. $\mathrm{N}$ Engl J Med 359: 895-905

13. Conde-Agudelo A, Romero R (2009) Antenatal magnesium sulfate for the prevention of cerebral palsy in preterm infants less than 34 weeks' gestation: a systematic review and metaanalysis. Am J Obstet Gynecol 200: 595-609.

14. Rouse DJ (2009) Magnesium sulfate for the prevention of cerebral palsy. Am J Obstet Gynecol 200: 610-612.

15. Doyle LW, Crowther CA, Middleton P, Marret S (2009) Antenatal magnesium sulfate and neurologic outcome in preterm infants: a systematic review. Obstet Gynecol 113: 1327-1333.

16. Coleman T, Cooper S, Thornton JG, Grainge MJ, Watts K, et al. (2012) A randomized trial of nicotine-replacement therapy patches in pregnancy. $\mathrm{N}$ Engl J Med 366: 808-818.

17. De Flora S, Izzotti A, D'Agostini F, Balansky RM (2001) Mechanisms of $\mathrm{N}$-acetylcysteine in the prevention of DNA damage and cancer, with special reference to smoking-related end-points. Carcinogenesis 22: 999-1013.

18. Van Schooten FJ, Besaratinia A, De Flora S, D'Agostini F, Izzotti A, et al. (2002) Effects of oral administration of $\mathrm{N}$-acetyl-L-cysteine: a multi-biomarker study in smokers. Cancer Epidemiol Biomarkers Prev 11: 167-175.

19. Swamy GK, Reddick KL, Brouwer RJ, Pollak KI, Myers ER (2011) Smoking prevalence in early pregnancy: comparison of self-report and anonymous urine cotinine testing. J Matern Fetal Neonatal Med 24: 86-90.

20. Tsuda H, Ohshima Y, Nomoto H, Fujita K, Matsuda E, et al. (2004) Cancer prevention by natural compounds. Drug Metab Pharmacokinet 19: 245-263.

21. Murphy M, Barraj L, Herman D, Bi X, Cheatham R, et al. (2012) Phytonutrien intake by adults in the United States in relation to fruit and vegetable consumption. J Acad Nutr Diet 112: 222-229.
22. Labonté MĖ, Cyr A, Baril-Gravel L, Royer MM, Lamarche B (2012) Validity and reproducibility of a web-based, self-administered food frequency questionnaire. Eur J Clin Nutr 66: 166-173.

23. Kay J, Chow WH, Chan TM, Lo SK, Kwok OH, et al. (2003) Acetylcysteine for prevention of acute deterioration of renal function following elective coronary angiography and intervention: a randomized controlled trial. JAMA 289: 553558 .

24. Ho E, Clarke JD, Dashwood RH (2009) Dietary sulforaphane, a histone deacetylase inhibitor for cancer prevention. J Nutr 139: 2393-2396.

25. Harms-Ringdahl M, Jenssen D, Haghdoost S (2012) Tomato juice intake suppressed serum concentration of 8 -oxodG after extensive physical activity. Nutr J 11: 29.

26. Ortsäter H, Grankvist N, Wolfram S, Kuehn N, Sjöholm A (2012) Die supplementation with green tea extract epigallocatechin gallate prevents progression to glucose intolerance in $\mathrm{db} / \mathrm{db}$ mice. Nutr Metab (Lond) 9: 11

27. Park SJ, Ahmad F, Philp A, Baar K, Williams T, et al. (2012) Resveratro ameliorates aging-related metabolic phenotypes by inhibiting CAMP phosphodiesterases. Cell 148: 421-433.

28. Tomé-Carneiro J, Gonzálvez M, Larrosa M, Yáñez-Gascón M, García-Almagro F, et al. (2012) One-year consumption of a grape nutraceutical containing resveratrol improves the inflammatory and fibrinolytic status of patients in primary prevention of cardiovascular disease. Am J Cardiol 110: 356-363.

29. Eroglu A, Hruszkewycz DP, dela Sena C, Narayanasamy S, Riedl KM, et al (2012) Naturally occurring eccentric cleavage products of provitamin A $\hat{i}^{2}$ carotene function as antagonists of retinoic acid receptors. J Biol Chem 287: 15886-15895.

30. Cowin P, Gold E, Aleksova J, O'Bryan M, Foster P, et al. (2010) Vinclozolin exposure in utero induces postpubertal prostatitis and reduces sperm production via a reversible hormone-regulated mechanism. Endocrinology 151 783-792.

31. Neal M, Mulligan Tuttle A, Casper R, Lagunov A, Foster W (2010) Ary hydrocarbon receptor antagonists attenuate the deleterious effects of benzo[a] pyrene on isolated rat follicle development. Reprod Biomed Online 21: 100108.

32. Forrest KY, Stuhldreher WL (2011) Prevalence and correlates of vitamin D deficiency in US adults. Nutr Res 31: 48-54

33. Tolppanen AM, Sayers A, Fraser WD, Lawlor DA (2012) Association of serum 25-hydroxyvitamin Dâ, $f$ and Dâ,, with academic performance in childhood: findings from a prospective birth cohort. J Epidemiol Community Health 66: 1137-1142.

34. Sørensen IM, Joner G, Jenum PA, Eskild A, Torjesen PA, et al. (2012) Maternal serum levels of 25-hydroxy-vitamin $D$ during pregnancy and risk of type diabetes in the offspring. Diabetes 61: 175-178.

35. Greenwald P (2002) Cancer prevention clinical trials. J Clin Oncol 20: 14S-22S. 